\title{
EUROfusion
}

EUROFUSION WPHCD-CP(16) 16620

Mr. Bader et al.

\section{Integrating a distributed antenna in DEMO: requirements and challenges}

Preprint of Paper to be submitted for publication in Proceedings of 29th Symposium on Fusion Technology (SOFT

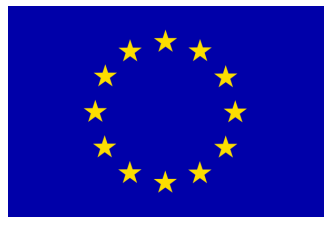

This work has been carried out within the framework of the EUROfusion Consortium and has received funding from the Euratom research and training programme 2014-2018 under grant agreement No 633053. The views and opinions expressed herein do not necessarily reflect those of the European Commission. 
This document is intended for publication in the open literature. It is made available on the clear understanding that it may not be further circulated and extracts or references may not be published prior to publication of the original when applicable, or without the consent of the Publications Officer, EUROfusion Programme Management Unit, Culham Science Centre, Abingdon, Oxon, OX14 3DB, UK or e-mail Publications.Officer@euro-fusion.org

Enquiries about Copyright and reproduction should be addressed to the Publications Officer, EUROfusion Programme Management Unit, Culham Science Centre, Abingdon, Oxon, OX14 3DB, UK or e-mail Publications.Officer@euro-fusion.org

The contents of this preprint and all other EUROfusion Preprints, Reports and Conference Papers are available to view online free at http://www.euro-fusionscipub.org. This site has full search facilities and e-mail alert options. In the JET specific papers the diagrams contained within the PDFs on this site are hyperlinked 


\title{
Integrating a distributed antenna in DEMO: requirements and challenges
}

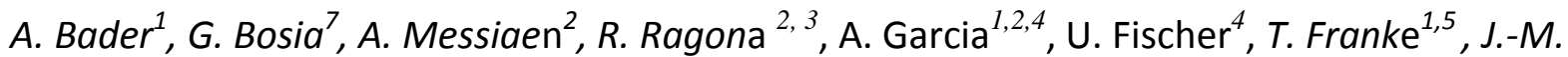 \\ Noterdaeme ${ }^{1,3}$, M.Q. $\operatorname{Tran}^{6}$, D. Van Eester ${ }^{2}$ \\ ${ }^{1}$ Max-Planck-Institut für Plasmaphysik, Boltzmannstr. 2, D-85748 Garching, Germany \\ ${ }^{2}$ LPP-ERM/KMS, B-1000 Brussels, Belgium \\ ${ }^{3}$ Applied Physics Department, Ghent University, B-9000 Gent, Belgium \\ ${ }^{4}$ Karlsruhe Institute of Technology (KIT), Kaiserstr. 12, 76131Karlsruhe, Germany \\ ${ }^{5}$ EUROfusion Consortium, Boltzmannstr. 2, D-85748 Garching, Germany \\ ${ }^{6}$ Swiss Plasma Center, Ecole Polytechnique Federale de Lausanne, 1015 Lausanne, Switzerland \\ ${ }^{7}$ University of Turin, Via Verdi, 8-10124, Turin, Italy
}

The use of efficient heating and current drive (H\&CD) systems is an important research priority for DEMO. The ion cyclotron range of frequency (ICRF) system is a promising candidate given decades of proven operational experience and low price equipment up to the MW level. ICRF antenna is presently situated in a port of limited size in the vacuum vessel (VV), which may be accompanied with operational limitations in DEMO, primarily because such configurations present high power density structures that can undergo malfunctions for the power levels anticipated for DEMO, because of voltage standoff limitations. To mitigate this, a novel ICRF antenna is under development and consists of toroidally distributed identical radiating straps integrated inside the blanket First Wall (FW). Given the antenna concept novelty and the strict requirements on the performance of DEMO, substantial design and integration efforts are needed. This paper highlights the requirements on the ICRF antenna and the challenges facing its integration in DEMO.

\section{Keywords: Ion cyclotron range of frequency (ICRF), distributed antenna, breeding blanket, tritium breeding ratio, blanket remote handling}

\section{Introduction}

The ICRF is an established method for H\&CD in several fusion facilities, and will be used in ITER for similar purposes [1]. ICRF is also one of the heating methods considered for DEMO.

ICRF antenna in its present configuration is shown in figure 1 and consists of few (or many, e.g. 24 for ITER) poloidal current straps, integrated in a port of limited size in the VV or within an antenna frame, in the machine. Such configuration carries with it a few drawbacks: (i) reduced coupling due to the evanescence of ICRF waves in the low density edge of plasma, (ii) possible high $\mathrm{Z}$ impurity release due to generation of larger sheath potentials due to $\mathrm{RF}$, resulting in accelerated ions producing surface sputtering, and (iii) possibility of voltage arcs or breakdown because high strap voltages and current amplitudes are needed to radiate the total required power, resulting in a high power density system. These drawbacks of present antenna configuration make the exploitation of ICRF increasingly challenging for DEMO. Alternatively, a novel ICRF concept is currently under development [2a,b] and $[4,5,6]$, constituted of identical poloidal current straps covering $360^{\circ}$ toroidally of the wall, as shown in figure $2 \mathrm{a}, \mathrm{b}$. Such antenna optimizes the coupling to the plasma because: (i) for a given inter-distance between the straps, the array coupling scales proportionally to the total strap number: this allows the coupling of large amount of power with affordable voltage and current constraints [4, 5], (ii) the absence of vertical septa between the straps leads to a significant increase of coupling with respect to a conventional antenna where the septa are inserted to reduce mutual coupling effects, and (iii) The radiated power spectrum can be very selective in the best $\mathrm{k} / /$ for coupling and heating $[5,6]$. Furthermore, such antenna potentially 
avoids, to a large extend, with its $360^{\circ}$ symmetry, the occurrence of induced currents at non-intended location, which would lead to RF electrical fields, sheaths and additional impurity production.

DEMO imposes stringent challenges on the integration on this novel antenna design not faced by its predecessor (e.g. ITER). The novel ICRF antenna will have to respect certain boundary conditions related to the breeding blanket (BB) such as not to impair its functions of neutron shielding and tritium breeding, being able to match the blanket modularity and have same power handling capabilities during operation as the blanket FW. The antenna must also be fully compatible with the remote handling $(\mathrm{RH})$ procedure for the blanket.

The configuration of the distributed antenna, suggested feeding concept, and the most significant points related to its integration in DEMO are highlighted in section 2.

\section{Distributed ICRF antenna configuration}

The novel distributed antenna is planned to be made of identical toroidal array sections of a limited poloidal extent, which can be contiguous to cover the complete wall periphery. Straps will be placed inside a recession and covered by the $\mathrm{FW}$, which will be slotted in the toroidal direction and acts as a Faraday Shield (FS). The dimensions of the recession and of straps are yet to be optimized, but typical presently suggested values are assumed: the recession will be $100 \mathrm{~cm}$ poloidally and $20 \mathrm{~cm}$ deep. Inside, there will be a ring array of $\sim 20 \mathrm{~cm}$ width straps (toroidal direction) separated by $\sim 3-4 \mathrm{~cm}$ each (the final separation is still to be defined.). The thickness of the antenna will be $\sim 2 \mathrm{~cm}$. For neutronics calculations a composition of antenna $10 \%+$ vacuum $90 \%$ is considered to be a good first assumption (the antenna is then considered as a mix of different layers of materials the straps may be made from, and any coolant material that may be used inside) [3]. Figure $2 a, b$ shows part of the antenna in one blanket sector. The antenna will be situated at a poloidal position between the vertical and equatorial ports. Final position will be influenced by a trade-off between physics functionality and engineering constraints.

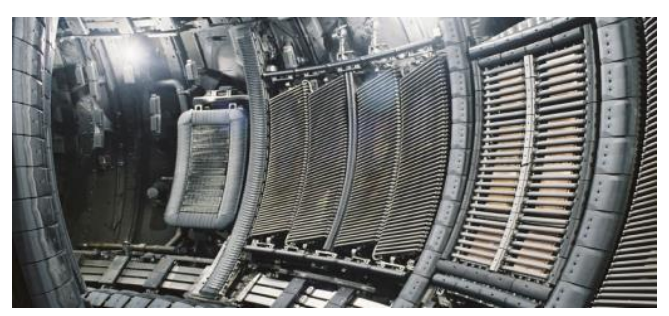

Fig.1. Example of present ICRF antenna configuration as seen on JET. On the right, the ITER Like Antenna (ILA), a high power density antenna, situated in a port of limited size toroidally and poloidally. In the middle, JET ICRF antenna, positioned in a frame inside the vessel. On the left is a Lower Hybrid (LH) antenna, not discussed here (figure adapted from [7]).

a)
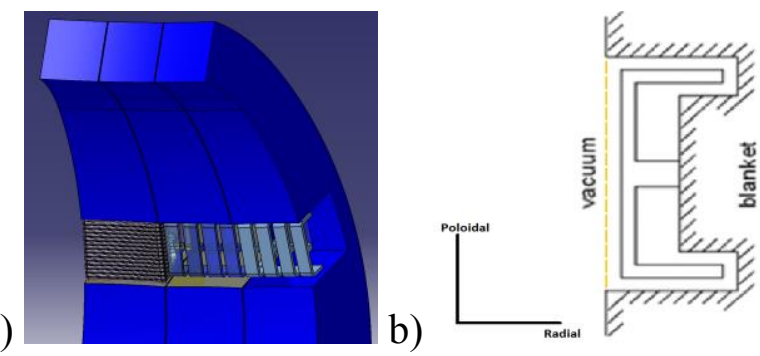

Fig.2a: View of ICRF antenna in one blanket sector (FS shown on left blanket segment for illustration, but exists on all segments); and $2 \boldsymbol{b}$ : radial build showing one strap integrated in the FW recession. Top and bottom parts of the strap form a capacitor configuration with the blanket, middle segment acts as a short circuit with the blanket. It also acts as a structural support and an inlet-outlet for the straps coolant.

\subsection{Antenna feeding}

One feeding concept discussed here, which avoids the necessity to feed and match each strap separately. Feeding is periodically done on sections of the array, with each blanket sector fed with a number of transmission lines (TLs) in a resonant ring configuration (figure 3) $[4,5,6]$. This system has many advantages: (i) only the first and last straps of each array section are connected to an external line, (ii) the generators remain matched for all loading conditions and is therefore load resilient. The actual feeding scheme, with the location of and number of feed lines to the straps to be fed per 
blanket sector is still under assessment. Possible approaches could be that for one blanket sector, two straps per central outboard blanket segment (COBS), cf. figure 3a, or (ii) the outermost straps in the left outboard blanket segment (LOBS) and right outboard blanket segment (ROBS) are fed as shown in figure $3 \mathrm{~b}$. The number of straps in each blanket sector must be chosen in order to avoid too large strap current decay between the first and last strap of each section [5]. The materials used for antenna straps will likely be similar to the blanket FW and $\mathrm{BB}$ structural materials itself, since, among other loads, it will receive the same neutron fluence. Exact materials composition will be decided based on ongoing integration and optimization studies. The feeding lines will have to be routed through the vertical ports down to the blanket segment where straps are located. Two lines are presently assumed to interface with each active (i.e. fed) strap, although one may also suffice. Optimum routing of lines via vertical ports needs to be assessed based on space available and neutron irradiation considerations. The materials of the feeding lines are likely the same as the BB structural material with a thin conducting layer (likely $\mathrm{Cu}$ ). The materials can be chosen to take into account the high temperature and neutron irradiation. Ceramic windows will present to separate the pressurized lines segments from those under vacuum. They can be installed behind dog-leg structures (of transmission line itself) and neutron shields. Another issue is the insulating material in the coaxial TLs, which position the inner conductor inside the outer conductor, and are made from ceramics. The choice of material type for the electrically insulating component and its location along TLs will be a trade-off between avoiding long lines under vacuum and potential loss of performance under the high neutron environment found in DEMO.

\section{Constraints imposed by DEMO}

Implementation of the novel ICRF antenna in DEMO may require laborious integration effort, and possible optimization of the initially assumed antenna configuration (described in section 2), in particular with the breeding blanket. The following is a description of the

primary integration issues of an ICRF antenna in DEMO. The points described are not meant as a comprehensive list that covers all integration related issues, but rather a highlight of those having prime priority at this initial design stage.

a)
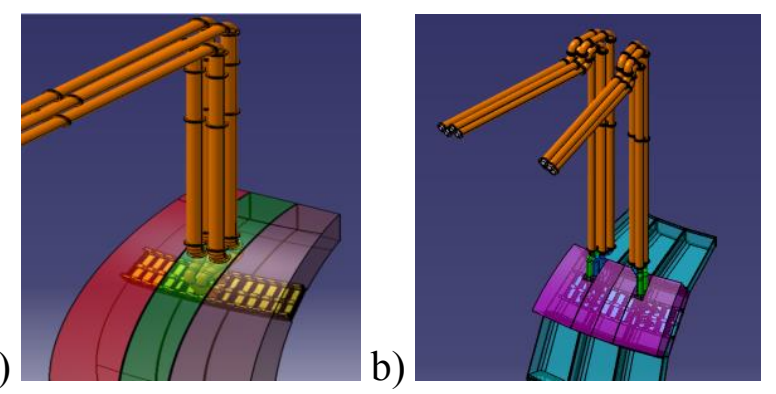

Fig.3a COBS feeding and $3 b$. ROBS and $L O B S$ feeding.

\subsection{Hosting breeding blanket}

Since the antenna is integrated in the blanket, it must ensure the tritium breeding and neutron shielding functions of the blanket are not hindered or degraded. Furthermore, the structure also should keep the complexity of the overall design at minimum, by e.g. sharing the cooling circuit with the blanket. Such requirements will have to be realized independent of which blanket type is eventually chosen for DEMO, though integration studies at this stage will focus on a single concept (likely the helium cooled pebble bed will be selected for initial integration studies).

DEMO is required to be a machine which ensures fuel self-sufficiency. Thus special attention was given to this issue in an earlier study [3] which investigated the effects of an ICRF antenna on the tritium breeding ratio (TBR), and provided an already encouraging case for the antenna integration in terms of TBR: with a total straps area of $70 \mathrm{~m}^{2}$, loss of TBR was equivalent to a $1.4 \mathrm{~m}^{2}$ opening in the $\mathrm{VV}$ equatorial plane, a factor of 50 less in area (namely, $\triangle \mathrm{TBR}$ does not exceed in the extreme cases (based on a parametric study) a value of 0.006). The TBR calculation will need to be refined when better definition of the antenna and its feeding becomes available (results reported in [3] do not, for instance, take the 
TLs that feed the straps into account). Another important factor related to the antenna integration in blanket is the structural integrity of the antenna. It must be ensured under both steady state and transient load conditions (e.g. plasma disruptions, vertical displacement events (VDE's), etc.).

\subsection{Remote handling compatibility}

Remote maintenance of the blanket is foreseen through the vertical ports following a specific process, where every blanket segment is treated separately from the others using remote handling equipment, and are lifted outside the VV for maintenance through DEMO's vertical ports. This is illustrated in figure 4 . From the antenna straps perspective, this requires that no toroidal connection between the straps should exist, and hence the blanket segments must remain modular. Additionally, the antenna must be as reliable as blanket FW itself, so that the reliability of the blankets is not reduced, and is desirable from RH perspective.. From The TLs perspective, the presently foreseen dimensions (12 or 9 inches in diameter) are considered challenging; hence an effort will be made to ensure further optimization of the TL size. Another requirement is to use TLs with long segments (known as large replaceable line segments) when possible, to reduce the number of maintained segments and hence potentially the overall maintenance time. Since part of the TLs will operate under vacuum by implementing ceramic windows, solutions for optimum placing of the windows along the TLs should be defined.
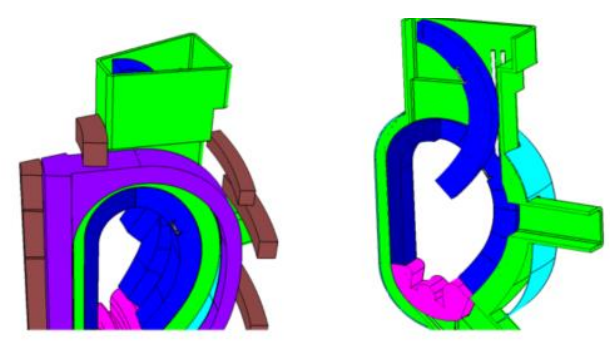

Fig.4.Remote handling of blanket segments is done through vertical ports. Each segment is handled separately, requiring no toroidal connections between them (and for ICRF antenna). Figure provided by EUROfusion's PPPT unit in Garching.

\section{Conclusions and future outlook}

This paper highlighted the necessity for a new ICRF system configuration for practical realization in DEMO. Focus is given on the distributed antenna. Any foreseen ICRF structure in DEMO needs to be compliant with other systems; of prime importance are the breeding blanket and remote handling procedures attached to it. Engineering design and integration of antenna is now an ongoing process, aiming to provide a consistent initial concept of an ICRF system in DEMO.

\section{Acknowledgment}

This work has been carried out within the framework of the EUROfusion Consortium and has received funding from the Euratom research and training programme 2014-2018 under grant agreement No 633053. Special thanks for the colleagues at KIT for supporting this work with Neutronic calculations.

\section{References}

[1] Lamalle, P.U., et al., Status of the ITER ICRF system design-'Externally Matched' approach; AIP Conf. Proc., 2011

[2a] Bosia, G., Low power density ion cyclotron arrays for fusion reactors; Fus. Eng. and Des. 92(2015)8-15

[2b] Bosia, G. and Ragona, R., Heating performances of IC in-blanket ring array; AIP Conf. Proc. 1689, 070001 (2015)

[3] Garcia, A., Effect on the Tritium Breeding Ratio for a distributed ICRF antenna in a DEMO reactor; Master thesis at the University of Polytechnique of Catalonia, 2015

[4] Ragona R. and Messiaen A. Nuclear Fusion, 56 (2016) 076009

[5] Messiaen A. and Ragona R. EPS conference Leuven (July 2016) paper P2.066

[6] Ragona R. and Messiaen A. SOFT conference Prague Sept. 2016

[7] https://www.euro-fusion.org/newsletter/astep-forward-in-heating-technology/ 